\title{
Impact of Annealing-induced Intermixing on the
}

\section{Electronic Level Alignment at the $\mathrm{In}_{2} \mathrm{~S}_{3} / \mathrm{Cu}(\mathrm{In}, \mathrm{Ga}) \mathrm{Se}_{2}$}

\section{Thin-film Solar Cell Interface}

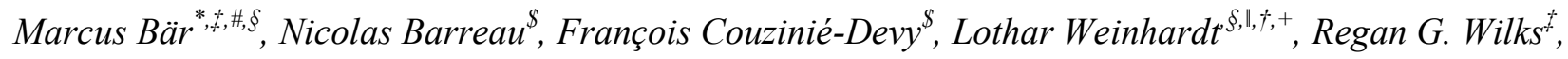
John Kessler ${ }^{\$}$, Clemens Heske,,,$\|,+,+$

${ }^{\star}$ Renewable Energy, Helmholtz-Zentrum Berlin für Materialien und Energie GmbH, Hahn-Meitner-Platz 1, 14109 Berlin, Germany

\# Institut für Physik und Chemie, Brandenburgische Technische Universität Cottbus-Senftenberg, Platz der Deutschen Einheit 1, 03046 Cottbus, Germany

$\S$ Department of Chemistry and Biochemistry, University of Nevada, Las Vegas (UNLV), 4505 Maryland Parkway, NV 89154-4003, USA

\$ Institut des Matériaux Jean Rouxel (IMN)-UMR 6502, Université de Nantes, CNRS, 2 rue de la Houssinière, BP 32229, 44322 Nantes Cedex 3, France

" Institute for Photon Science and Synchrotron Radiation, Karlsruhe Institute of Technology, Hermann-v.Helmholtz-Platz 1, 76344 Eggenstein-Leopoldshafen, Germany 
${ }^{\dagger}$ ANKA Synchrotron Radiation Facility, Karlsruhe Institute of Technology, 76344 EggensteinLeopoldshafen, Germany

${ }^{+}$Institute for Chemical Technology and Polymer Chemistry, Karlsruhe Institute of Technology,

Engesserstr. 18/20, 76128 Karlsruhe, Germany

\section{Corresponding Author}

*Email: marcus.baer@helmholtz-berlin.de 
For a detailed discussion of the x-ray photoelectron spectroscopy (XPS) and x-ray emission spectroscopy (XES) data and the derived chemical interface structure see Refs. ${ }^{1,2}$ To determine the band alignment of the heavily intermixed $\mathrm{In}_{2} \mathrm{~S}_{3} / \mathrm{Cu}(\mathrm{In}, \mathrm{Ga}) \mathrm{Se}_{2}$ interface, it is crucial to test for interface-induced changes in band bending (iibb) at the absorber surface, as well as a possible band bending in the buffer layer. For the heavily intermixed materials system under investigation, this presents a particular challenge. At a chemically abrupt interface, the change in band bending upon interface formation is monitored by the binding energy shifts in the core levels related to the bottom (absorber) and top (buffer) layer. As core levels could also shift due to changes in chemical environment, any chemical interaction induced by the formation of the buffer/absorber interface (as observed here) complicates the determination of such band bending corrections. To identify core level candidates that can be unambiguously assigned to the substrate, we note that Ga does not appear to be involved in the observed interfacial intermixing/interdiffusion processes (see Refs. 1 and 2). Furthermore, we find a significant reduction of the Se signal as well, ${ }^{1,2}$ suggesting that it also might serve well as a "substrate signal", but in this case it cannot entirely be ruled out that the Se atoms that are replaced by $\mathrm{S}$ atoms at the (former) absorber surface also diffuse into the overlayer. To derive data-based insights into these questions, the positions of the Ga $2 \mathrm{p}_{3 / 2}$ and $\mathrm{Se} 3 \mathrm{~d}_{5 / 2}$ photoemission lines for all samples in the series were determined by a fit analysis and are displayed in Table S1. We find unusually large variations in core-level binding energies; for the thin overlayers, both core levels shift downwards (Se: $\sim 0.1 \mathrm{eV}, \mathrm{Ga}: \sim 0.3 \mathrm{eV}$ ). For the 1/16 sample, for which the two signals can still be evaluated, we find an additional upward shift (Se: $\sim 0.2 \mathrm{eV}, \mathrm{Ga}: \sim 0.1 \mathrm{eV}$ ), while the fit analysis for thicker overlayers is questionable due to the low signal. We argue that the overall shift (i.e., between the CIGSe sample and the 1/16 sample) of the Ga core level $(-0.2 \pm 0.1 \mathrm{eV})$ is the most reliable shift to be used for the interfaceinduced band bending analysis for the following reasons. First, we speculate that the surface composition of the $1 / 64$ and 1/32 samples is expected to be more strongly influenced by $\mathrm{Cu}$ diffusion and hence was found to be significantly different from the samples with thicker 
overlayer. ${ }^{1}$ This has an impact on the core level positions of the overlayer and - due to a potentially different interface-induced band bending - also of the substrate, leading to an artificial initial shift that should not be incorporated in the determination of the proper iibb. Second, as mentioned above, we expect some Se atoms to be displaced by the $\mathrm{S}$ atoms at the (former) CIGSe surface, thus possibly leading to a line position of the (rather weak) Se line of $1 / 16$ sample that is no longer representative of the former absorber surface. Finally, we note that a small, additional downward band bending upon buffer deposition is in line with previous observations at the $\mathrm{CdS} / \mathrm{CIGSe}$ interface and the fact that high efficiencies are achieved with the annealing-induced intermixing of the $\mathrm{In}_{2} \mathrm{~S}_{3} / \mathrm{CIGSe}$ system.

Furthermore, due to the fact that both the bottom and top layer contains indium and that sulfur diffuses into the upper region of the absorber layer, ${ }^{1,2}$ an unambiguous assignment of core level binding energies to (exclusively) the overlayer is not possible for very thin overlayer samples. To account for this issue, band bending corrections can only be determined using samples in which the $\mathrm{S}$ and In signals from the absorber (and the $\mathrm{S}$ signal from the $\mathrm{Na}_{\mathrm{x}} \mathrm{S}$ ) only give a negligible contribution to the overall $\mathrm{S}$ and In core level spectra (as judged from the attenuation of the Ga $2 p$ and Se $3 \mathrm{~d}$ lines, as well as the $\mathrm{Na} / \mathrm{In}$ ratio - see Ref. 1 for details). This is the case for the 1/16 sample and all thicker overlayers. Comparing the $\mathrm{S} 2 \mathrm{~s}$ and $\mathrm{In} 3 \mathrm{~d}_{3 / 2}$ core level positions for the 1/16 and 1/1 samples (Table S1), it can be observed that the band edges in the buffer layer shift downwards by $(-0.1 \pm 0.1) \mathrm{eV}$ towards the buffer/absorber interface. 
Table S1. Core level peak BE positions of all samples, as well as their relative shifts that have been considered for the determination of the interface-induced band bending correction. Error bars for the core level peak positions are $\pm 0.05 \mathrm{eV}$. Values given in parentheses are not included in the considerations due to low signal intensity and potentially different chemical/electronic interface structure (in the case of Se and $\mathrm{Ga}$ ) or (in the case of $\mathrm{S}$ and $\mathrm{In}$ ) due to their assignment to CIGSe and CIGSSe${ }^{[1]}$ (rather than to the overlayer). The core level binding energies that have been used to compute the shift of the band edges for the $1 / 16 \mathrm{In}_{2} \mathrm{~S}_{3} /$ CIGSe samples due to interface formation are shown in bold.

\begin{tabular}{|c|c|c|c|c|}
\hline BE $(e V)$ & $\operatorname{Se} 3 d_{5 / 2}$ & Ga $2 p_{3 / 2}$ & S 2s & In $3 d_{3 / 2}$ \\
\hline CIGSe & 54.18 & 1118.00 & - & $(452.33)$ \\
\hline $1 / 64 \mathrm{In}_{2} \mathrm{~S}_{3} / \mathrm{CIGSe}$ & $(54.26)$ & $(1118.39)$ & $(226.29)$ & $(452.60)$ \\
\hline $1 / 32 \mathrm{In}_{2} \mathrm{~S}_{3} / \mathrm{CIGSe}$ & $(54.28)$ & $(1118.31)$ & $(226.28)$ & $(452.63)$ \\
\hline 1/16 $\operatorname{In}_{2} \mathrm{~S}_{3} / \mathrm{CIGSe}$ & 54.07 & 1118.23 & 225.87 & 452.39 \\
\hline $1 / 8 \mathrm{In}_{2} \mathrm{~S}_{3} / \mathrm{CIGSe}$ & $(54.10)$ & $(1118.00)$ & 225.79 & 452.15 \\
\hline $1 / 4 \mathrm{In}_{2} \mathrm{~S}_{3} / \mathrm{CIGSe}$ & - & - & 225.80 & 452.21 \\
\hline $1 / 2 \mathrm{In}_{2} \mathrm{~S}_{3} / \mathrm{CIGSe}$ & - & - & 225.84 & 452.37 \\
\hline 1/1 $\mathrm{In}_{2} \mathrm{~S}_{\mathbf{3}} / \mathrm{CIGSe}$ & - & - & 225.77 & 452.27 \\
\hline $\begin{array}{c}\text { Shift } \\
\text { (for } 1 / 16 \mathrm{In}_{2} \mathrm{~S}_{3} / \mathrm{CIGSe} \\
( \pm \mathbf{0 . 1} \mathbf{e V})\end{array}$ & -0.1 & -0.2 & -0.1 & -0.1 \\
\hline
\end{tabular}

References

(1) Bär, M.; Barreau, N.; Couzinié-Devy, F.; Pookpanratana, S.; Klaer, J.; Blum, M.; Zhang, Y.; Yang, W.; Denlinger, J. D.; Schock, H.-W.; Weinhardt, L.; Kessler, J.; Heske, C. Nondestructive Depthresolved Spectroscopic Investigation of the Heavily Intermixed $\operatorname{In}_{2} \mathrm{~S}_{3} / \mathrm{Cu}(\mathrm{In}, \mathrm{Ga}) \mathrm{Se}_{2}$ Interface. Appl. Phys. Lett. 2010, 96, 184101.

(2) Bär, M.; Barreau, N.; Couzinié-Devy, F.; Félix, R.; Klaer, J.; Pookpanratana, S.; Blum, M.; Zhang, Y.; Denlinger, J. D.; Yang, W.; Wilks, R. G.; Weinhardt, L.; Schock, H.-W.; Kessler, J.; Heske, C. The Heavily Intermixed $\operatorname{In}_{2} \mathrm{~S}_{3} / \mathrm{Cu}(\mathrm{In}, \mathrm{Ga}) \mathrm{Se}_{2}$ Interfaces Revealed by Photoelectron and Soft X-ray Emission Spectroscopy. Proc. 39th IEEE Photovoltaic Specialists Conference, Tampa, FL, June 16$21,2013,857-862$. 